\title{
General Info: International e-Conference on Bioengineering for Health and Environment (ICBHE 2020)
}

\section{Organized by}

Department of Biotechnology, School of Bio \& Chemical Engineering, Centre for International Relations, Sathyabama Institute of Science \& Technology, India

School of Bioscience, Faculty of Medicine, Bioscience and Nursing, MAHSA University, Malaysia.

* Correspondence: antonysamrot@gmail.com; raji.naomi10@gmail.com

Conference highlights

\begin{tabular}{|c|c|}
\hline Biotechnology & Bioinformatics \\
\hline ㅁ Plant Biotechnology & New Generation Bioinformatics \\
\hline a Stem Cells \& Cancer Research & a Big DATA Analysis \\
\hline advances in Clinical Biochemistry & Structural Bioinformatics \\
\hline a Biopharmaceuticals & a Bioinformatics \& Proteomics \\
\hline د Nanobiotechnology & ] Bioinformatics \& Genomics \\
\hline a Industrial Microbiology & व Bioinformatics Algorithms \& \\
\hline 口 Drug Delivery & Databases \\
\hline animal Biotechnology & a Bioinformatics in Clinical Case \\
\hline a Environmental Biotechnology & Reports \\
\hline Industrial Microbiology \& & Bioinformatics in Drug Discovery \\
\hline Biotechnology & a System Biology \\
\hline Chemical Engineering & Biomedical Engineering \\
\hline a Biochemical Engineering for & a Biomaterials \\
\hline Environment & a Recent Advances in Biomedical \\
\hline ه Biofuels & Engineering \\
\hline a Modeling of Environmental & a Biomedical Instrumentation \\
\hline Processes & \\
\hline Waste water treatment & \\
\hline
\end{tabular}

Keynote speakers

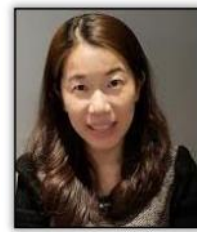

Dr. Kai-Yin Lo, Associate Professor Department of Agricultural Chemistry, National Taiwan University, $1 \mathrm{Sec} .4$,

Roosevelt Road, Taipei 10617, Taiwan.

Dr. Suresh Kumar, Associate Professor Department of Medical Microbiology and Parasitology University Putra Malaysia, Jalan University, Serdang, 43400 Seri Kembangan, Selangor, Malaysia.
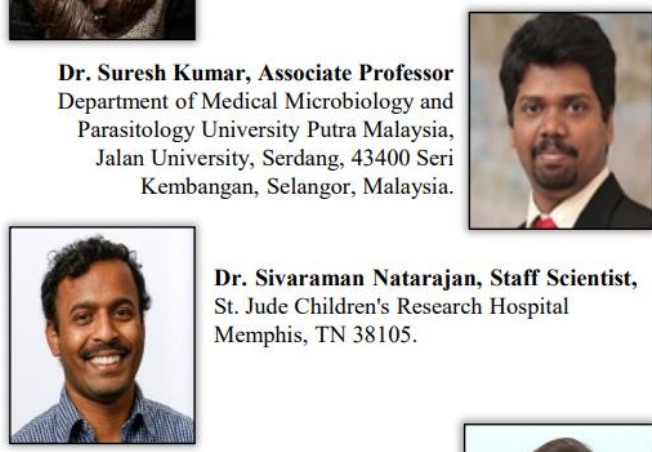

Dr. Sivaraman Natarajan, Staff Scientist, St. Jude Children's Research Hospital Memphis, TN 38105

Dr. Sameera Sastry Panchangan, Post Doctoral Fellow, Southern Methodist University, Dallas, Texas, USA. 


\section{Patrons}

Dr. Mariazeena Johnson, Chancellor, Sathyabama

Dr. Marie Johnson, President, Sathyabama

Prof. Tan Sri Datuk Dr. HJ Mohamed Haniffa bin HJ Abdullah, Pro-Chancellor \& Executive Chairman, MAHSA

Dato' Dr. Shahril bin Prof. Tan Sri Datuk Dr. Haji Mohamed Haniffa, MAHSA

Dato' Sharon bin Prof. Tan Sri Datuk Dr. Haji Mohamad Haniffa, MAHSA

\section{Advisors}

Dr. T. Sasipraba, Vice Chancellor, Sathyabama

Prof. Dato' Dr. Ishak bin Abdul Razak, Vice Chancellor, MAHSA

Dr. Wilson Aruni, Pro-Vice Chancellor, Sathyabama

Dr.E. Logashanmugam, Pro-Vice Chancellor, Sathyabama

Dr. S. S. Rau, Registrar, Sathyabama

Dr. Ganesh Ramachandran, Dean, FOMBN, MAHSA

\section{Organizing secretaries}

Dr. T. Sasipraba, Vice Chancellor, Sathyabama

Dr. Wilson Aruni, Pro-Vice Chancellor, Sathyabama

Dr. Paulraj Ponnaiah, Deputy Dean, MAHSA

Dr. Antony V Samrot, MAHSA

\section{Convenors}

Dr. Ramesh Kumar V, Head, Biotechnology

Mr. Narendrakumar G, Head, Biotechnology

Ms. Raji P, Assistant Professor, Sathyabama

Ms. Ketharin A/P Tunasamy, Head, School of Bioscience, MAHSA

(C) 2020 by the authors. This article is an open-access article distributed under the terms and conditions of the Creative Commons Attribution (CC BY) license (https://creativecommons.org/licenses/by/4.0/).
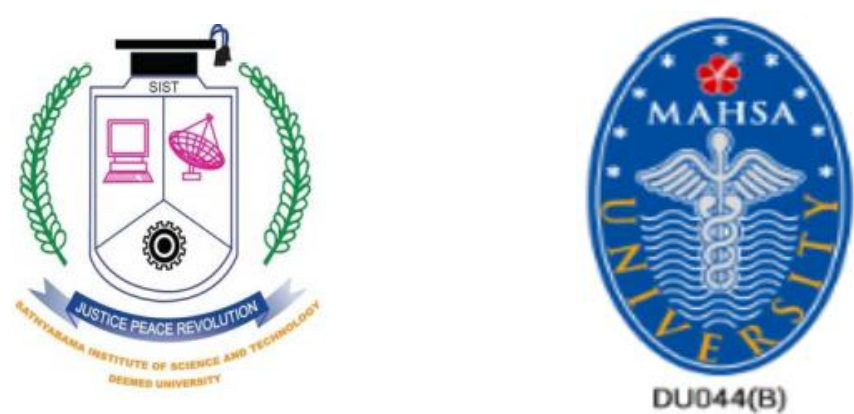